\section{Review Article \\ Gastroenterology \& Hepatology}

Check for updates

\title{
Common Locations of Gastric Cancer: Review of Research from the Endoscopic Submucosal Dissection Era
}

\author{
Su Jin Kim (1) and Cheol Woong Choi (1)
}

Department of Internal Medicine, Pusan National University School of Medicine and Research Institute for Convergence of Biomedical Science and Technology, Pusan National University Yangsan Hospital, Yangsan, Korea

\section{OPEN ACCESS}

Received: Apr 1, 2019

Accepted: Aug 2, 2019

Address for Correspondence: Cheol Woong Choi, MD, PhD

Department of Internal Medicine, Medical Research Institute, Pusan National University School of Medicine and Research Institute for Convergence of Biomedical Science and Technology, Pusan National University Yangsan Hospital, 20 Geumo-ro,

Mulgeum-eup, Yangsan 50612

Republic of Korea.

E-mail: luckyace@hanmail.net

(C) 2019 The Korean Academy of Medical Sciences.

This is an Open Access article distributed under the terms of the Creative Commons Attribution Non-Commercial License (https:// creativecommons.org/licenses/by-nc/4.0/) which permits unrestricted non-commercial use, distribution, and reproduction in any medium, provided the original work is properly cited.

ORCID iDs

Su Jin Kim (iD

https://orcid.org/0000-0003-3816-9664 Cheol Woong Choi (iD

https://orcid.org/0000-0001-8867-3039

Disclosure

The authors have no potential conflicts of interest to disclose.

\section{Author Contributions}

Conceptualization: Choi CW. Writing original draft: Kim SJ. Writing - review \& editing: Choi CW.

\section{ABSTRACT}

Detection of early-stage gastric cancer improves the prognosis of patients. Endoscopic submucosal dissection (ESD) is a curative and stomach-preserving treatment for early gastric cancer (EGC) associated with a low risk of lymph node metastasis. However, several studies have reported missed diagnosis of gastric cancer. Therefore, endoscopists are required to learn accurate diagnostic skills to eliminate endoscopic blind spots. A systematic screening protocol to map the entire stomach without blind spots reduces the risk of missed lesions. Knowledge of the features of EGC or dysplasia is essential to identify suspicious lesion. Information of the common sites of occurrence of EGC can also enable a detailed endoscopic examination to improve detection rates. Previous reports investigating the location of gastric cancers resected by ESD or surgery showed that the antrum and lesser curvature of stomach were predominantly affected. Helicobacter pylori-induced atrophic changes advance from the antrum to the corpus along the lesser curvature, predominantly affecting these areas. Gastric cancers in the antrum and the lower corpus are also commonly missed during screening examination. Therefore, a careful examination of the lower third stomach is warranted to avoid missing synchronous and metachronous gastric lesions. Knowledge of the location of EGC enables accurate endoscopic examination and detection of EGC in early stage.

Keywords: Gastric Cancer; Location; Endoscopy

\section{INTRODUCTION}

Gastric cancer is a major global health issue, particularly in Asian countries including Korea, Japan, and China. ${ }^{1}$ Despite advances in endoscopic and surgical resection equipment and techniques, gastric cancer is the third leading cause of cancer mortality globally. Detection of gastric cancer in the early stages and prompt treatment are associated with favorable prognosis. ${ }^{2,3}$ Patients diagnosed with early gastric cancer (EGC) during screening endoscopy show low risk of lymph node metastasis and can undergo endoscopic submucosal dissection (ESD) as curative local treatment for stomach preservation. However, most patients with EGC present with non-specific symptoms. Previous studies have shown that the prevalence of advanced gastric cancer was higher in patients undergoing diagnostic examination without previous screening endoscopy to investigate the causes of gastrointestinal symptoms. ${ }^{4}$ 
Thus, a mass screening program for gastric cancer has been adopted in Korea and Japan. A recent study evaluated the effect of gastric cancer screening via biennial upper endoscopy or gastrography in adults aged $\geq 40$ years, which is the Korean National Cancer Screening Program. This study reported the effectiveness of such assessment in reducing the gastric cancer-related mortality risk. ${ }^{4}$ However, there is a report about the incidence of interval gastric cancer (IGC) in patients who received the periodic endoscopic examination in Korea. ${ }^{5}$ Therefore, meticulous endoscopic examination is mandatory to reduce false-negative rates.

Several factors should be considered to optimize the diagnostic yield of endoscopy. First, the administration of antispasmodics and mucolytic agents such as pronase facilitates a careful examination as the pretreatment. ${ }^{6}$ Second, adequate air insufflation and removal of mucus for optimal visualization of the mucosal surface are essential for accurate detection of EGC as technical aspect of examination. Third, a systematic screening protocol to map the entire stomach without blind spots reduces the risk of missed lesions as the observation procedure. ${ }^{7}$ In addition to a technically accurate endoscopic procedure, knowledge of the features of suspicious lesions is important to detect EGC. Key signs of EGC are lesions with an irregular surface (elevated or depressed lesions) and color (reddish or discolored) changes, and spontaneous bleeding (Fig. 1). If EGC or dysplasia is suspected, the chromoendoscopy using indigo carmine can be helpful to define the surface morphology of suspicious lesions. Finally, advanced imaging techniques such as magnifying endoscopy with narrow band image provide detailed information regarding small gastric lesions. This review discusses EGC locations detected from screening and surveillance endoscopy, especially in Korea. This article will contribute significantly to the literature by providing a better understanding of EGC and will guide endoscopists in performing careful endoscopic examination and surveillance.

\section{INTRAGASTRIC LOCATION OF EGC INDICATIVE ESD}

Mucosal cancers that on histopathological examination measure $<2 \mathrm{~cm}$ in size and show well-differentiated features without ulceration can be effectively treated by ESD owing to their low risk of lymph node metastasis. ${ }^{8} \mathrm{~A}$ recent multicenter single-arm confirmatory trial (JCOG0607) performed by the Japan Clinical Oncology Group suggested that ESD should be the standard treatment (preferred over gastrectomy) for intestinal-type EGC that fulfills the expanded indications. ${ }^{9}$ For undifferentiated EGC sized less than $2 \mathrm{~cm}$ without ulceration, a meta-analysis including 972 undifferentiated type EGC from 14 retrospective studies reported that the ESD showed favorable short-term outcome. ${ }^{10}$ Although long-term data after ESD are further needed to confirm the safety and efficacy, the endoscopic resection has
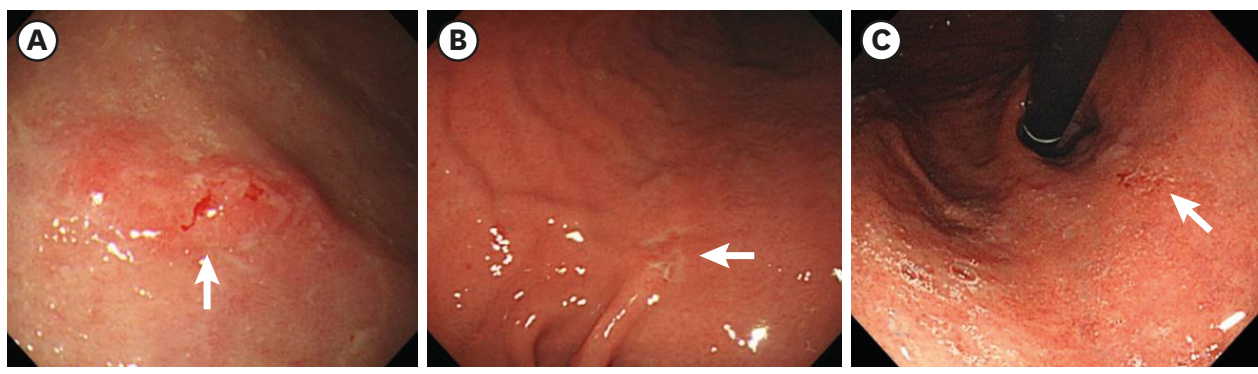

Fig. 1. Images of upper endoscopy for early gastric cancers. (A) Elevated and reddish lesion sized $1.5 \mathrm{~cm}$ with spontaneous bleeding (arrow) is found on the antrum greater curvature. (B) Depressed and discolored lesion sized $1.0 \mathrm{~cm}$ is found on the lower body lesser curvature. (C) Flat lesion sized $0.8 \mathrm{~cm}$ with inhomogeneous erythema is found on the cardia. 
been performed for the curative intention in clinical practice. ${ }^{11}$ However, endoscopists should consider that the curative resection rate is lower than that of ESD for differentiated type EGC.12

Early detection of EGC is important to achieve curative resection through ESD.

Several retrospective studies conducted in Korea demonstrated that the lower part is the most common site to perform ESD for the treatment of EGC (Table 1). One study in which 647 consecutive EGC lesions were resected by ESD also showed that the most frequent site of occurrence of EGC was the lower part of the stomach (89.6\%). This study has reported that the lesser curvature was the most common site of involvement (43.6\%). ${ }^{13}$

The lower part of the stomach includes the antrum-pylorus and the lower third of the stomach corpus including the angle. The mid-to-upper part refers to the middle and upper thirds of the stomach corpus including the cardia and the fundus. H. pylori infection causes gastric mucosal inflammation. Intestinal-type gastric cancer is preceded by an inflammationinduced cascade of precancerous lesions induced by inflammation (the atrophy-metaplasiadysplasia-adenocarcinoma sequence). ${ }^{14}$ Atrophic mucosal changes advance from the antrum to the corpus along the lesser curvature. ${ }^{15}$ The distribution pattern of atrophy explains the higher incidence of EGC in the lower parts and the lesser curvature of the stomach. Although the inflammation-induced carcinogenesis cascade by $H$. pylori infection is not the primary cause of undifferentiated type gastric cancer, it is speculated that inflammation itself promotes development of these gastric cancers. Therefore, the undifferentiated-type EGC commonly occurs in the lower portions of the stomach.13,16

\section{CHARACTERISTICS OF SURGICALLY RESECTED GASTRIC CANCER BASED ON ITS LOCATION}

Various studies performed in Korea showed that the lower part is the most frequent site to detect EGC in patients who received the surgical resection (Table 2). One study evaluated 313 consecutive surgically resected small gastric cancers measuring $<2 \mathrm{~cm}$ in size, to obtain information regarding the common sites of origin of this type of gastric cancer. ${ }^{17}$ EGCs

Table 1. Locations of early gastric cancer that received endoscopic resection

\begin{tabular}{|c|c|c|c|c|}
\hline Author & Year & No. & Study design & Tumor location (\%) \\
\hline \multirow[t]{5}{*}{ Min et al.36 } & 2015 & 1,497 & Single center & Body (21.8) \\
\hline & & & & Fundus (21.8) \\
\hline & & & & Cardia (21.8) \\
\hline & & & & Antrum (78.2) \\
\hline & & & & Angle (78.2) \\
\hline \multirow[t]{3}{*}{ Kim et al. ${ }^{3}$} & 2015 & 165 & Single center & Upper (6.1) \\
\hline & & & & Middle (14.5) \\
\hline & & & & Lower (79.4) \\
\hline \multirow[t]{3}{*}{ Choi et al. 37} & 2015 & 961 & Single center & Upper (6.0) \\
\hline & & & & Middle (21.2) \\
\hline & & & & Lower (72.8) \\
\hline \multirow[t]{3}{*}{ Ahn et al.38 } & 2011 & 1,370 & Single center & Upper (7.1) \\
\hline & & & & Middle (32.3) \\
\hline & & & & Lower (60.6) \\
\hline \multirow[t]{3}{*}{ Shin et al.39 } & 2015 & 1,105 & Multicenter & Upper (4.3) \\
\hline & & & & Middle (28.5) \\
\hline & & & & Lower (67.2) \\
\hline
\end{tabular}




\begin{tabular}{|c|c|c|c|c|}
\hline Author & Year & No. & Study design & Tumor location (\%) \\
\hline \multirow[t]{3}{*}{ Jeon et al.40 } & 2018 & 275 & Single center & Upper (4.4) \\
\hline & & & & Middle (33.1) \\
\hline & & & & Lower (62.5) \\
\hline \multirow[t]{3}{*}{ Kim et al. 3} & 2015 & 292 & Single center & Upper (7.2) \\
\hline & & & & Middle (14.4) \\
\hline & & & & Lower (78.4) \\
\hline \multirow[t]{3}{*}{ Ryu et al.41 } & 2016 & 144 & Multicenter & Upper (4.9) \\
\hline & & & & Middle (32.9) \\
\hline & & & & Lower (62.2) \\
\hline \multirow[t]{3}{*}{ Hahn et al.42 } & 2018 & 1,206 & Multicenter & Upper (8.3) \\
\hline & & & & Middle (32.8) \\
\hline & & & & Lower (58.9) \\
\hline
\end{tabular}

were primarily detected in the antrum-angle (42.2\%) and the cardia (26.2\%). Most (68\%) small gastric cancers originated along the lesser curvature. This result concurs with a study showing EGC resected by ESD. Undifferentiated EGCs occur more commonly in the distal stomach than the proximal site. 18,19

Clinicopathological studies comparing early adenocarcinoma of the gastric cardia and early adenocarcinoma of the distal stomach have revealed that proximal gastric cancer shows the following characteristics: 1) elderly male predominance, 2) greater tendency toward submucosal invasion and, 2) better histopathological differentiation. ${ }^{20,21}$ In these studies, proximal gastric cancer was defined as a tumor with its epicenter located within $3 \mathrm{~cm}$ below the gastroesophageal junction (to avoid misclassification of esophageal cancer as gastric cancer). These reports would help endoscopists to investigate the antrum-angularis and the lesser curvature more intensively, as well as to evaluate the cardia more closely in elderly men in geographical areas with a high prevalence of $H$. pylori infection. Notably, endoscopic examination in young women with a family history of gastric cancer needs to focus on the gastric corpus, particularly the area near the greater curvature. ${ }^{22}$

\section{CHARACTERISTICS OF MISSED SYNCHRONOUS GASTRIC LESIONS}

Several reports have described missed synchronous gastric cancer after ESD or gastrectomy.23,24 The delayed detection of synchronous lesions may lead to an additional treatment including ESD or surgery that can result in patient's discomfort, and increased medical cost.

Early detection of synchronous gastric lesions before these progress to invasive cancers is important to improve the prognosis of patients. A meta-analysis including 22 studies (six studies conducted in Korea) reported that the rate of missing synchronous lesions after ESD was $9.0 \% .{ }^{25}$ An excessive focus on primary gastric neoplasms during endoscopy can result in that other synchronous lesions might be missed. ${ }^{26}$ One study reported that more than half of cases with missed synchronous lesion had images of missed lesion captured during the previous endoscopy examination. ${ }^{27}$ The lower parts of the stomach is the most frequent location to find missed synchronous lesions (Table 3). Factors associated with missed synchronous lesions were origin in the small size, and non-elevated morphology.23,28 Nonelevated and small synchronous lesions are likely to be missed because these are difficult to detect on endoscopy. Therefore, endoscopists should consider the possibility of synchronous 
Table 3. Locations of missed synchronous gastric lesions after endoscopic resection in Korea

\begin{tabular}{lcccc}
\hline Author & Year & No. & Study design & Tumor location (\%) \\
\hline Nam et al.26 & 2018 & 77 & Single center & Upper (5.9) \\
& & & & $\begin{array}{c}\text { Middle (21.3) } \\
\text { Lower (72.7) }\end{array}$ \\
Yoo et al.28 & 2013 & 29 & Single center & Upper (6.9) \\
& & & & Middle (31.0) \\
& & & & Lower (62.1) \\
Lee et al.24 & 2010 & 12 & Single center & Upper (6.3) \\
& & & & Middle (43.8) \\
& 2017 & & & Lower (50.0) \\
Kim et al.43 & 141 & Multicenter & Upper (7.1) \\
& & & Middle (32.6) \\
& & & Lower (60.3) \\
\hline
\end{tabular}

gastric neoplasms and carefully examine the entire stomach to evaluate not only primary lesions, but also check for synchronous lesions.

\section{CHARACTERISTICS OF METACHRONOUS GASTRIC CANCER (MGC)}

MGC is generally defined as a gastric cancer that developed at a location distant from the primary resection site during follow-up endoscopy after index ESD. ${ }^{29}$ Retrospective studies have reported that incidence of metachronous lesions ranged from 3.6\% to $6.1 \% .30,31$ Therefore, endoscopic surveillance should be continued after gastric ESD. In a retrospective study including 2,779 patients who received ESD for EGC and endoscopic surveillance in Korea, the 5 and 10 year cumulative incidence of MGC or high grade dysplasia were $4.7 \%$ and $11.3 \% .{ }^{30}$ Lower third of the stomach was the most common location for metachronous lesions (Table 4). Other features of MGC are small, differentiated intramucosal cancers sized less than $2 \mathrm{~cm} .{ }^{29}$ Therefore, the regular endoscopic surveillance can allow repeat endoscopic resection for MGC.

\section{CHARACTERISTICS OF IGC}

The high detection rate of EGC in Korea and Japan is attributable to regular screening endoscopy performed in these countries ${ }^{4}$; however, several studies have reported cases of IGC. 25,32 The concept of IGC is derived from colon cancer and refers to cancer that is diagnosed between the time of screening and the next scheduled screening endoscopy. Therefore, this term is applicable only in countries such as Korea and Japan where a national screening program for gastric cancer is operational. A Korean study that evaluated the clinicopathological characteristics of IGC reported that the incidence of IGC diagnosed

Table 4. Locations of metachronous gastric lesions after endoscopic resection in Korea

\begin{tabular}{lcccc}
\hline Author & Year & No. & Study design & Tumor location (\%) \\
\hline Cho et al.30 & 2017 & 96 & Single center & Upper (10.4) \\
& & & Middle (18.8) \\
& & & Lower (70.8) \\
Lee et al.44 & 2018 & \multirow{2}{*}{100} & Multicenter & Upper (7) \\
& & & Middle (37) \\
& & & Lower (56) \\
\hline
\end{tabular}


between the time of screening and post-screening endoscopy was $2.0 \%\left(16 / 81,762\right.$ subjects). ${ }^{5}$ Characteristics of IGC were cancer detected in the lower corpus of the stomach $(70.6 \%)$ and histopathological evidence of undifferentiated lesions (70.6\%). The doubling time of gastric cancer is approximately $2-3$ years. ${ }^{33}$ Therefore, most IGC lesions in this study could be lesions that progressed from missed gastric mucosal abnormalities. A meta-analysis investigating the rate of missed gastric cancer during upper endoscopy showed a 9.4\% incidence of missed gastric cancer. Identification of cancer within an interval of 6 months to 3.5 years after a negative endoscopy was defined as missed cancer. Thus, the incidence of missed lesions may be higher. ${ }^{25}$ Most missed gastric cancers were poorly differentiated lesions located in the gastric antrum and lower body. 34 This result concurs with a previous study describing IGC. The anatomical variations in different parts of the stomach contribute to missed gastric cancer. Small-sized gastric cancer lesions occurring in the corpus might be obscured within the gastric folds. Frequent belching is associated with a difficult examination of the stomach corpus. ${ }^{35}$

\section{CONCLUSION}

Early detection improves the prognosis and reduces the economic burden of gastric cancer. Additionally, ESD is curative treatment for stomach preservation in such patients when EGC has not progressed to a stage where ESD is not feasible. Sound knowledge of standard endoscopic techniques and endoscopic features of EGC are important to improve the detection rate of EGC. Therefore, endoscopists need appropriate training to improve their technical skills and knowledge of various gastric lesions. This article focuses on the importance of understanding the common sites of occurrence of EGC as a clue for the accurate detection of gastric cancer in the early stages. The lesser curvature and the lower part of the stomach were observed to be the predominant locations affected by EGCs that are resectable by ESD or gastrectomy. The incidence of gastric cancer affecting the cardia was higher in elderly men. Most IGCs were poorly differentiated on histopathological examination and were located in the gastric antrum and lower body. Endoscopists should be mindful of the possibility of non-elevated synchronous lesions. Finally, newly detected EGC and MGC are frequently found in the lower third, and the majority of MGCs found during endoscopic surveillance are small, differentiated intramucosal cancer. In conclusion, knowledge regarding the location of EGC is useful for accurate endoscopic examination for screening and surveillance.

\section{REFERENCES}

1. Bray F, Ferlay J, Soerjomataram I, Siegel RL, Torre LA, Jemal A. Global cancer statistics 2018: GLOBOCAN estimates of incidence and mortality worldwide for 36 cancers in 185 countries. CA Cancer J Clin 2018;68(6):394-424. PUBMED | CROSSREF

2. Kikuchi S, Katada N, Sakuramoto S, Kobayashi N, Shimao H, Watanabe M, et al. Survival after surgical treatment of early gastric cancer: surgical techniques and long-term survival. Langenbecks Arch Surg 2004;389(2):69-74. PUBMED | CROSSREF

3. Kim YI, Kim YW, Choi IJ, Kim CG, Lee JY, Cho SJ, et al. Long-term survival after endoscopic resection versus surgery in early gastric cancers. Endoscopy 2015;47(4):293-301. PUBMED | CROSSREF 
4. Jun JK, Choi KS, Lee HY, Suh M, Park B, Song SH, et al. Effectiveness of the Korean National Cancer Screening Program in reducing gastric cancer mortality. Gastroenterology 2017;152(6):1319-1328.e7. PUBMED | CROSSREF

5. Park MS, Yoon JY, Chung HS, Lee H, Park JC, Shin SK, et al. Clinicopathologic characteristics of interval gastric cancer in Korea. Gut Liver 2015;9(2):166-73.

PUBMED | CROSSREF

6. Kim GH, Cho YK, Cha JM, Lee SY, Chung IK. Efforts to increase image quality during endoscopy: the role of pronase. World J Gastrointest Endosc 2016;8(5):267-72. PUBMED | CROSSREF

7. Yao K. The endoscopic diagnosis of early gastric cancer. Ann Gastroenterol 2013;26(1):11-22. PUBMED

8. Japanese Gastric Cancer Association. Japanese classification of gastric carcinoma: 3rd English edition. Gastric Cancer 2011;14(2):101-12.

PUBMED | CROSSREF

9. Hasuike N, Ono H, Boku N, Mizusawa J, Takizawa K, Fukuda H, et al. A non-randomized confirmatory trial of an expanded indication for endoscopic submucosal dissection for intestinal-type gastric cancer (cT1a): the Japan Clinical Oncology Group study (JCOG0607). Gastric Cancer 2018;21(1):114-23. PUBMED | CROSSREF

10. Bang CS, Baik GH, Shin IS, Kim JB, Suk KT, Yoon JH, et al. Endoscopic submucosal dissection for early gastric cancer with undifferentiated-type histology: a meta-analysis. World J Gastroenterol 2015;21(19):6032-43. PUBMED | CROSSREF

11. Kim JH. Important considerations when contemplating endoscopic resection of undifferentiated-type early gastric cancer. World J Gastroenterol 2016;22(3):1172-8.

PUBMED | CROSSREF

12. Jeon HK, Lee SJ, Kim GH, Park DY, Lee BE, Song GA. Endoscopic submucosal dissection for undifferentiated-type early gastric cancer: short- and long-term outcomes. Surg Endosc 2018;32(4):1963-70. PUBMED | CROSSREF

13. Kang DH, Choi CW, Kim HW, Park SB, Kim SJ, Nam HS, et al. Location characteristics of early gastric cancer treated with endoscopic submucosal dissection. Surg Endosc 2017;31(11):4673-9. PUBMED | CROSSREF

14. Correa P. A human model of gastric carcinogenesis. Cancer Res 1988;48(13):3554-60. PUBMED

15. Kimura K, Satoh K, Ido K, Taniguchi Y, Takimoto T, Takemoto T. Gastritis in the Japanese stomach. Scand J Gastroenterol Suppl 1996;214:17-20. PUBMED | CROSSREF

16. Kang HY, Kim SG, Kim JS, Jung HC, Song IS. Clinical outcomes of endoscopic submucosal dissection for undifferentiated early gastric cancer. Surg Endosc 2010;24(3):509-16. PUBMED | CROSSREF

17. Huang Q, Shi J, Sun Q, Gold JS, Chen J, Wu H, et al. Clinicopathological characterisation of small ( $2 \mathrm{~cm}$ or less) proximal and distal gastric carcinomas in a Chinese population. Pathology 2015;47(6):526-32. PUBMED | CROSSREF

18. Ye BD, Kim SG, Lee JY, Kim JS, Yang HK, Kim WH, et al. Predictive factors for lymph node metastasis and endoscopic treatment strategies for undifferentiated early gastric cancer. J Gastroenterol Hepatol 2008;23(1):46-50. PUBMED

19. Park YD, Chung YJ, Chung HY, Yu W, Bae HI, Jeon SW, et al. Factors related to lymph node metastasis and the feasibility of endoscopic mucosal resection for treating poorly differentiated adenocarcinoma of the stomach. Endoscopy 2008;40(1):7-10. PUBMED | CROSSREF

20. Huang Q, Fang C, Shi J, Sun Q, Wu H, Gold JS, et al. Differences in clinicopathology of early gastric carcinoma between proximal and distal location in 438 chinese patients. Sci Rep 2015;5(1):13439. PUBMED | CROSSREF

21. Tajima Y, Nakanishi Y, Yoshino T, Kokawa A, Kusano M, Shimoda T. Clinicopathological study of early adenocarcinoma of the gastric cardia: comparison with early adenocarcinoma of the distal stomach and esophagus. Oncology 2001;61(1):1-9. PUBMED | CROSSREF

22. Bai Y, Li ZS. Endoscopic, clinicopathological features and prognosis of very young patients with gastric cancer. J Gastroenterol Hepatol 2011;26(11):1626-9.

PUBMED | CROSSREF 
23. Kato M, Nishida T, Yamamoto K, Hayashi S, Kitamura S, Yabuta T, et al. Scheduled endoscopic surveillance controls secondary cancer after curative endoscopic resection for early gastric cancer: a multicentre retrospective cohort study by Osaka University ESD study group. Gut 2013;62(10):1425-32. PUBMED | CROSSREF

24. Lee HL, Eun CS, Lee OY, Han DS, Yoon BC, Choi HS, et al. When do we miss synchronous gastric neoplasms with endoscopy? Gastrointest Endosc 2010;71(7):1159-65.

PUBMED | CROSSREF

25. Pimenta-Melo AR, Monteiro-Soares M, Libânio D, Dinis-Ribeiro M. Missing rate for gastric cancer during upper gastrointestinal endoscopy: a systematic review and meta-analysis. Eur J Gastroenterol Hepatol 2016;28(9):1041-9. PUBMED | CROSSREF

26. Nam HS, Kim HW, Choi CW, Kang DH, Park SB, Kim SJ, et al. Characteristics of overlooked synchronous gastric epithelial neoplasia after endoscopic submucosal dissection. Medicine (Baltimore) 2018;97(39):e12536. PUBMED | CROSSREF

27. Gong EJ, Lee JH, Jung K, Cho CJ, Na HK, Ahn JY, et al. Characteristics of missed simultaneous gastric lesions based on double-check analysis of the endoscopic image. Clin Endosc 2017;50(3):261-9. PUBMED | CROSSREF

28. Yoo JH, Shin SJ, Lee KM, Choi JM, Wi JO, Kim DH, et al. How can we predict the presence of missed synchronous lesions after endoscopic submucosal dissection for early gastric cancers or gastric adenomas? J Clin Gastroenterol 2013;47(2):e17-22. PUBMED | CROSSREF

29. Abe S, Oda I, Minagawa T, Sekiguchi M, Nonaka S, Suzuki H, et al. Metachronous gastric cancer following curative endoscopic resection of early gastric cancer. Clin Endosc 2018;51(3):253-9. PUBMED | CROSSREF

30. Cho CJ, Ahn JY, Jung HY, Jung K, Oh HY, Na HK, et al. The incidence and locational predilection of metachronous tumors after endoscopic resection of high-grade dysplasia and early gastric cancer. Surg Endosc 2017;31(1):389-97. PUBMED | CROSSREF

31. Hosokawa O, Kaizaki Y, Watanabe K, Hattori M, Douden K, Hayashi H, et al. Endoscopic surveillance for gastric remnant cancer after early cancer surgery. Endoscopy 2002;34(6):469-73. PUBMED | CROSSREF

32. Chadwick G, Groene O, Riley S, Hardwick R, Crosby T, Hoare J, et al. Gastric cancers missed during endoscopy in England. Clin Gastroenterol Hepatol 2015;13(7):1264-1270.e1. PUBMED | CROSSREF

33. Fujita S. Biology of early gastric carcinoma. Pathol Res Pract 1978;163(4):297-309. PUBMED | CROSSREF

34. Cho YS, Chung IK, Kim JH, Jung Y, Lee TH, Park SH, et al. Risk factors of developing interval early gastric cancer after negative endoscopy. Dig Dis Sci 2015;60(4):936-43. PUBMED | CROSSREF

35. Lee SP, Sung IK, Kim JH, Lee SY, Park HS, Shim CS. Factors impacting patient cooperation during elective gastroscopy. Korean J Intern Med (Korean Assoc Intern Med) 2017;32(5):819-26. PUBMED | CROSSREF

36. Min BH, Kim ER, Kim KM, Park CK, Lee JH, Rhee PL, et al. Surveillance strategy based on the incidence and patterns of recurrence after curative endoscopic submucosal dissection for early gastric cancer. Endoscopy 2015;47(9):784-93. PUBMED | CROSSREF

37. Choi J, Kim SG, Im JP, Kim JS, Jung HC. Long-term clinical outcomes of endoscopic resection for early gastric cancer. Surg Endosc 2015;29(5):1223-30. PUBMED | CROSSREF

38. Ahn JY, Jung HY, Choi KD, Choi JY, Kim MY, Lee JH, et al. Endoscopic and oncologic outcomes after endoscopic resection for early gastric cancer: 1370 cases of absolute and extended indications. Gastrointest Endosc 2011;74(3):485-93.

PUBMED | CROSSREF

39. Shin KY, Jeon SW, Cho KB, Park KS, Kim ES, Park CK, et al. Clinical outcomes of the endoscopic submucosal dissection of early gastric cancer are comparable between absolute and new expanded criteria. Gut Liver 2015;9(2):181-7.

PUBMED | CROSSREF 
40. Jeon HK, Kim GH, Lee BE, Park DY, Song GA, Kim DH, et al. Long-term outcome of endoscopic submucosal dissection is comparable to that of surgery for early gastric cancer: a propensity-matched analysis. Gastric Cancer 2018;21(1):133-43. PUBMED | CROSSREF

41. Ryu SJ, Kim BW, Kim BG, Kim JH, Kim JS, Kim JI, et al. Endoscopic submucosal dissection versus surgical resection for early gastric cancer: a retrospective multicenter study on immediate and long-term outcome over 5 years. Surg Endosc 2016;30(12):5283-9.

PUBMED | CROSSREF

42. Hahn KY, Park CH, Lee YK, Chung H, Park JC, Shin SK, et al. Comparative study between endoscopic submucosal dissection and surgery in patients with early gastric cancer. Surg Endosc 2018;32(1):73-86. PUBMED | CROSSREF

43. Kim HH, Kim JH, Kim GH, Choi MG, Jee SR, Song GA. Causes of missed synchronous gastric epithelial neoplasms with endoscopic submucosal dissection: a multicenter study. Scand J Gastroenterol 2013;48(11):1339-46.

PUBMED | CROSSREF

44. Lee HJ, Lee YJ, Lee JY, Kim ES, Chung WJ, Jang BK, et al. Characteristics of synchronous and metachronous multiple gastric tumors after endoscopic submucosal dissection of early gastric neoplasm. Clin Endosc 2018;51(3):266-73.

PUBMED | CROSSREF 